Pacific

Journal of

Mathematics

\title{
BOUNDARY ASYMPTOTICAL BEHAVIOR OF LARGE SOLUTIONS TO HESSIAN EQUATIONS
}

YONG HUANG 


\title{
BOUNDARY ASYMPTOTICAL BEHAVIOR OF LARGE SOLUTIONS TO HESSIAN EQUATIONS
}

\author{
YONG HUANG
}

\begin{abstract}
We consider the exact asymptotic behavior of smooth solutions to boundary blow-up problems for the $k$-Hessian equation on $\Omega$, where $\partial \Omega$ is strictly $(k-1)$-convex. Similar results were obtained by Cîrstea and Trombetti when $k=n$ (the Monge-Ampère equation) and by Bandle and Marcus for a semilinear equation.
\end{abstract}

\section{Introduction and main results}

We investigate the qualitative properties of solutions to the boundary blow-up problem for the $k$-Hessian equation of the form

$$
\begin{cases}H_{k}\left[D^{2} u\right]=\sigma_{k}\left(\lambda_{1}, \ldots, \lambda_{n}\right)=b(x) f(u), & x \in \Omega, \\ u(x)=\infty, & x \in \partial \Omega,\end{cases}
$$

where $b(x)$ is a continuous weight function, $\lambda_{1}, \ldots, \lambda_{n}$ are eigenvalues of $D^{2} u$, the Hessian matrix of a $C^{2}$-function $u$ defined over $\Omega$, and $\Omega$ is a bounded domain in $\mathbb{R}^{n}$. The boundary condition means $u(x) \rightarrow+\infty$ as $d(x) \triangleq \operatorname{dist}(x, \partial \Omega) \rightarrow 0_{+}$.

Following [Caffarelli et al. 1985; Trudinger 1995], $\sigma_{k}$ is defined by

$$
\sigma_{k}\left(\lambda_{1}, \ldots, \lambda_{n}\right)=\sum_{1 \leq i_{1}<\cdots<i_{k} \leq n} \lambda_{i_{1}} \cdots \lambda_{i_{k}} .
$$

One can solve (1-1) in a class of $k$-convex functions by [Caffarelli et al. 1985; Jian 2006]. Recall that a function $u \in C^{2}(\Omega)$ is called $k$-convex (or strictly $k$-convex) if $\left(\lambda_{1}, \ldots, \lambda_{n}\right) \in \bar{\Gamma}_{k}$ (or $\left.\left(\lambda_{1}, \ldots, \lambda_{n}\right) \in \Gamma_{k}\right)$ for every $x \in \Omega$, where $\Gamma_{k}$ is the convex cone with vertex at the origin given by

$$
\Gamma_{k}=\left\{\lambda=\left(\lambda_{1}, \ldots, \lambda_{n}\right) \in \mathbb{R}^{n} \mid \sigma_{j}(\lambda)>0, j=1, \ldots, k\right\} .
$$

Obviously,

$$
\Gamma_{1} \supset \Gamma_{2} \supset \cdots \supset \Gamma_{n}=\left\{\lambda=\left(\lambda_{1}, \ldots, \lambda_{n}\right) \in \mathbb{R}^{n} \mid \lambda_{j}>0, j=1, \ldots, k\right\},
$$

MSC2000: primary 35J60; secondary 35B40.

Keywords: boundary blow-up problem, Hessian equations.

The first author is supported by Tianyuan Fund of Mathematics (10826060). 
where $\Gamma_{n}$ is the positive cone, and $\sigma_{k}\left(\lambda_{1}, \ldots, \lambda_{n}\right)$ is elliptic in the class of $k$-convex functions.

For an open bounded subset $\Omega$ of $\mathbb{R}^{n}$ with boundary of class $C^{2}$ and for every $x \in \partial \Omega$, we denote by $\rho_{1}(x), \ldots, \rho_{n-1}(x)$ the principal curvatures of $\partial \Omega$ (relative to the interior normal). Recall that $\Omega$ is said to be $l$-convex if $\left(\rho_{1}(x), \ldots, \rho_{n-1}(x)\right) \in$ $\bar{\Gamma}_{l}$, and it is called strictly $l$-convex if $\left(\rho_{1}(x), \ldots, \rho_{n-1}(x)\right) \in \Gamma_{l}$, for every $x \in \partial \Omega$. In particular, strictly $(n-1)$-convex is just strictly convex.

Using radial function methods and techniques of ordinary differential inequality, Jian [2006] constructed various barriers functions, then proved existence and nonexistence theorems using those barriers. Furthermore, generic boundary blowup rates for the solution are derived for the $k$-Hessian equation with boundary blow-up problem. In this paper, we derive accurately the blow-up rate of solutions to boundary blow-up problems for Hessian equations.

Let $\mathfrak{K}_{\ell}$ denote the set of all positive nondecreasing $C^{1}$-functions $m$ defined on $(0, v)$, for some $v>0$, for which there exists

$$
\lim _{t \rightarrow 0^{+}} \frac{\int_{0}^{t} m(s) d s}{m(t)}=0 \text { and } \lim _{t \rightarrow 0^{+}} \frac{d}{d t}\left(\frac{\int_{0}^{t} m(s) d s}{m(t)}\right)=\ell .
$$

A complete characterization of $\mathfrak{K}_{\ell}$ (according to $\ell \neq 0$ or $\ell=0$ ) is provided by [Cîrstea and Rădulescu 2006].

One has the following examples for special $\ell$, where $p>0$ is arbitrary:

(a) $m(t)=(-1 / \ln t)^{p}$ with $\ell=1$,

(b) $m(t)=t^{p}$ with $\ell=1 /(p+1)$,

(c) $m(t)=e^{-1 / t^{p}}$ with $\ell=0$.

Definition 1.1. A positive measurable function $f$ defined on $[a, \infty)$, for some $a>0$, is called regularly varying at infinity with index $q$, written $f \in \mathbb{R} \mathbb{V}_{q}$, if for each $\lambda>0$ and some $q \in \mathbb{R}$,

$$
\lim _{t \rightarrow \infty} \frac{f(\lambda t)}{f(t)}=\lambda^{q}
$$

The real number $q$ is called the index of regular variation.

When $q=0$, we have:

Definition 1.2. A positive measurable function $L$ defined on $[a, \infty)$, for some $a>0$, is called regularly varying at infinity, if for each $\lambda>0$ and some $q \in \mathbb{R}$,

$$
\lim _{t \rightarrow \infty} \frac{L(\lambda t)}{L(t)}=1
$$

By Definitions 1.1 and 1.2, if $f \in \mathbb{R} \mathbb{V}_{q}$, it can be represented in the form

$$
f(t)=u^{q} L(t) .
$$


Notation. If $H$ is a nondecreasing function on $\mathbb{R}$, then we denote by $H^{\leftarrow}$ the (left-continuous) inverse of $H$ [Resnick 1987], that is,

$$
H^{\leftarrow}(y)=\inf \{s: H(s) \geq y\}
$$

If $\alpha>0$ is sufficiently large, we define

$$
\mathscr{P}(u)=\sup \left\{\frac{f(y)}{y^{k}}: \alpha \leq y \leq u\right\}, \quad \text { for } u \geq \alpha .
$$

Problem (1-1) is the Laplace operator when $k=1$. There are many papers resolving existence, uniqueness and asymptotic behavior issues for blow-up solutions of semilinear/quasilinear elliptic equations: for instance [Osserman 1957; Resnick 1987; Véron 1992; Bandle and Marcus 1992; 1995; García-Melián et al. 2001; Chuaqui et al. 2004; Cîrstea and Rădulescu 2006; García-Melián 2006].

When $k=n$, problem (1-1) is the Monge-Ampère equation, for which Cîrstea and Trombetti [2008] obtained existence, uniqueness and asymptotic behavior; see also [Guan and Jian 2004; Mohammed 2007].

The boundary blow-up problem of the $k$-Hessian equation was considered in [Salani 1998; Colesanti et al. 2000; Jian 2006]. See also [Takimoto 2006] for recent results on boundary blow-up problems for $k$-curvature equations, where there is a considerable difference between the cases $1 \leq k \leq n-1$ and $k=n$. However, we can unify them by using techniques from [Colesanti et al. 2000; Cîrstea and Trombetti 2008] for $k$-Hessian equations.

Our asymptotic results are obtained in the case when $\partial \Omega$ is strictly $(k-1)$ convex, but for $k$-curvature equations in [Cîrstea and Trombetti 2008], the condition that $\partial \Omega$ is strictly convex is needed.

Theorem 1.3. Let $n \geq 2$ and $\Omega$ be a smooth, strictly ( $k-1)$-convex bounded domain in $\mathbb{R}^{n}$. Assume that $f \in \mathbb{R} \mathbb{V}_{q}$ with $q>k$ and there exists $m \in \mathfrak{K}_{\ell}$ such that

$$
0<\beta^{-}=\liminf _{d(x) \rightarrow 0} \frac{b(x)}{m^{k+1}(d(x))} \quad \text { and } \quad \limsup _{d(x) \rightarrow 0} \frac{b(x)}{m^{k+1}(d(x))}=\beta^{+}<\infty .
$$

Then, every $k$-convex blow-up solution $u_{\infty}$ of (1-1) satisfies

$$
\xi^{-} \leq \liminf _{d(x) \rightarrow 0} \frac{u}{\phi(d(x))} \quad \text { and } \quad \limsup _{d(x) \rightarrow 0} \frac{u}{\phi(d(x))} \leq \xi^{+},
$$

where $\phi$ is defined by

$$
\phi(t)=\mathscr{P} \leftarrow\left(\left(\int_{0}^{t} m(s) d s\right)^{-k-1}\right), \quad \text { for } t>0 \text { small },
$$


and $\xi^{ \pm}$are positive constants given by

$$
\frac{\left(\xi^{+}\right)^{k-q}}{\beta^{-}} \max _{\partial \Omega} \sigma_{k-1}=\frac{\left(\xi^{-}\right)^{k-q}}{\beta^{+}} \min _{\partial \Omega} \sigma_{k-1}=\frac{((q-k) /(n+1))^{k+1}}{1+\ell(q-k) /(k+1)} .
$$

On the other hand, Colesanti et al. [2000] established asymptotic estimates for the behavior of the smallest viscosity solution near the boundary of $\Omega$ for the Hessian equation

$$
\begin{cases}H_{k}\left[D^{2} u\right]=f(u), & x \in \Omega, \\ u(x)=\infty, & x \in \partial \Omega .\end{cases}
$$

Theorem 1.3 may also been seen as a generalization of the asymptotic behavior for the viscosity solution in [Colesanti et al. 2000].

Remark 1.4. In the setting of Theorem 1.3, $\lim _{d(x) \rightarrow 0} u / \phi(d(x))$ exists provided that $\Omega$ is a ball and (1-8) holds with $\beta^{-}=\beta^{+} \in(0, \infty)$. The latter condition is equivalent to saying that

$$
b(x) \sim(m(d(x)))^{k+1} \quad \text { as } d(x) \rightarrow 0, \quad \text { for some } m \in \mathfrak{K}_{\ell} .
$$

More exactly, when $\Omega$ is a ball of radius $R>0$, Theorem 1.3 reads as follows.

Corollary 1.5. Let $\Omega=B_{R}$ be a ball of radius $R>0$ and $f \in \mathbb{R} \mathbb{V}_{q}$ with $q>k$. If (1-13) holds, then every strictly $k$-convex blow up solution $u$ of (1-1) satisfies

$$
u(x) \sim \xi \phi(d(x)) \quad \text { as } d(x) \rightarrow 0,
$$

where $\phi$ is defined by (1-10) and $\xi$ is given by

$$
\xi=\left(\frac{((q-k) /(k+1))^{k+1} R^{k-1}}{1+\ell(q-k) /(k+1)}\right)^{1 /(k-q)} .
$$

Under slightly more restrictive conditions than those in Theorem 1.3, there is at most one strictly $k$-convex blow-up solution of (1-1).

Theorem 1.6. Let $\Omega$ be a smooth, strictly (k-1)-convex, bounded domain in $\mathbb{R}^{n}$. Suppose $f \in \mathbb{R} \mathbb{V}_{q}$ with $q>k$, and $f(u) / u^{k}$ is increasing on $(0, \infty)$. Then, (1-1) has at most one strictly $k$-convex blow-up solution, provided that either

(i) $b$ is positive on $\bar{\Omega}$, or

(ii) $b$ is zero on $\partial \Omega, \Omega$ is a ball of radius $R>0$ and (1-13) holds.

Remark 1.7. When $k=n$ (the Monge-Ampère equation), Theorems 1.3 and 1.6 were obtained in [Cîrstea and Trombetti 2008]. 


\section{Preliminaries}

Proposition 2.1. Let $\Omega$ be an open subset of $\mathbb{R}^{n}$ with $n \geq 2$. If $h \in C^{2}(\mathbb{R})$ and $g \in C^{2}(\Omega)$ then

$$
\begin{aligned}
\sigma_{k}\left(D^{2} h(g(x))\right)=\left(h^{\prime}(g(x))\right)^{k-1} h^{\prime \prime} & (x) \sigma_{k-1}\left(\left.D^{2} g\right|_{i, j}\right) g_{i} g_{j} \\
& +\left(h^{\prime}(g(x))\right)^{k} \sigma_{k}\left(D^{2} g\right), \quad \text { for all } x \in \Omega,
\end{aligned}
$$

where $\left.D^{2} g\right|_{i, j}$ is the cofactor of the $(i, j)$-th entry of the symmetric matrix $D^{2} g(x)$.

For $\mu>0$, we set $\Gamma_{\mu}=\{x \in \bar{\Omega}: d(x, \partial \Omega)<\mu\}$.

Remark 2.2. If $\Omega$ is bounded and $\partial \Omega \in C^{l}$ for $l \geq 2$, then there exists a positive constant $\mu$ depending on $\Omega$ such that $d \in C^{l}\left(\Gamma_{\mu}\right)$. (See also Lemma 14.16 in [Gilbarg and Trudinger 1998].)

Corollary 2.3. Let $\Omega$ be bounded with $\partial \Omega \in C^{l}$ for $l \geq 2$. Assume that $\mu>0$ is small such that $d \in C^{2}\left(\Gamma_{\mu}\right)$ and $h$ is a $C^{2}$-function on $(0, \mu)$. Let $x_{0} \in \Gamma_{\mu} \backslash \partial \Omega$ and $y_{0} \in \partial \Omega$ be such that $\left|x_{0}-y_{0}\right|=d\left(x_{0}\right)$. Then, we have

$$
\begin{aligned}
\sigma_{k}\left(D^{2} h\left(d\left(x_{0}\right)\right)\right)=\left(-h^{\prime}\left(d\left(x_{0}\right)\right)\right)^{k-1} h^{\prime \prime}( & \left.d\left(x_{0}\right)\right) \sigma_{k-1}\left(\varepsilon_{1}, \ldots, \varepsilon_{n-1}\right) \\
& +\left(-h^{\prime}\left(d\left(x_{0}\right)\right)\right)^{k} \sigma_{k}\left(\varepsilon_{1}, \ldots, \varepsilon_{n-1}\right),
\end{aligned}
$$

where $\rho_{1}\left(y_{0}\right), \ldots, \rho_{n-1}\left(y_{0}\right)$ are the principal curvatures of $\partial \Omega$ at $y_{0}$ and $\varepsilon_{i}=$ $\rho_{i}\left(y_{0}\right) /\left(1-\rho_{i}\left(y_{0}\right) d\left(x_{0}\right)\right), i=1, \ldots, n-1$.

Proof. It is easy to calculate that the expression of the Hessian matrix of $d$ at $x_{0}$ in terms of a principal coordinate system at $y_{0}$ (see also Lemma 14.17 in [Gilbarg and Trudinger 1998]), namely

$$
\begin{aligned}
D^{2} d\left(x_{0}\right) & =\operatorname{diag}\left(\frac{-\rho_{1}\left(y_{0}\right)}{1-\rho_{1}\left(y_{0}\right) d\left(x_{0}\right)}, \ldots, \frac{-\rho_{n-1}\left(y_{0}\right)}{1-\rho_{n-1}\left(y_{0}\right) d\left(x_{0}\right)}, 0\right), \\
\operatorname{Dd}\left(x_{0}\right) & =(0, \ldots, 0,1) .
\end{aligned}
$$

Thus by Proposition 2.1, we obtain

$$
\begin{aligned}
& \sigma_{k}\left(D^{2} h\left(d\left(x_{0}\right)\right)\right) \\
& =\left(-h^{\prime}\left(d\left(x_{0}\right)\right)\right)^{k-1} h^{\prime \prime}\left(d\left(x_{0}\right)\right) \sigma_{k-1}\left(\left[\begin{array}{ccc}
\frac{\rho_{1}\left(y_{0}\right)}{1-\rho_{1}\left(y_{0}\right) d\left(x_{0}\right)} & \\
& \ddots & \\
& \frac{\rho_{n-1}\left(y_{0}\right)}{1-\rho_{n-1}\left(y_{0}\right) d\left(x_{0}\right)}
\end{array}\right]\right) \\
& \quad+\left(-h^{\prime}\left(d\left(x_{0}\right)\right)\right)^{k} \sigma_{k}\left(\left[\begin{array}{ccc}
\frac{\rho_{1}\left(y_{0}\right)}{1-\rho_{1}\left(y_{0}\right) d\left(x_{0}\right)} & \\
& \ddots & \\
& & \frac{\rho_{n-1}\left(y_{0}\right)}{1-\rho_{n-1}\left(y_{0}\right) d\left(x_{0}\right)}
\end{array}\right]\right) \cdot \square
\end{aligned}
$$


We now give a brief account of the definitions and properties of regularly varying functions; see also [Resnick 1987; Cîrstea and Trombetti 2008].

Proposition 2.4 (Uniform convergence theorem). If $L$ is slowly varying, $\frac{L(\lambda u)}{L(u)}$ tends to 1 as $u \rightarrow \infty$, uniformly on each compact $\lambda$-set in $(0, \infty)$.

Proposition 2.5. (See also Proposition 4.9 in [Cîrstea and Trombetti 2008].)

(i) If $R \in \mathbb{R V}_{q}$, then $\lim _{u \rightarrow \infty} \log R(u) / \log u=q$.

(ii) If $R_{1} \in \mathbb{R} V_{q_{1}}$ and $R_{2} \in \mathbb{R} \mathbb{q}_{q_{2}}$ with $\lim _{u \rightarrow \infty} R_{2}(u)=\infty$, then

$$
R_{1} \circ R_{2} \in \mathbb{R} \mathbb{V}_{q_{1} q_{2}} \text {. }
$$

(iii) Suppose $R$ is nondecreasing and $R \in \mathbb{R V}_{q}, 0<q<\infty$. Then

$$
R^{\leftarrow} \in \mathbb{R V}_{q^{-1}}
$$

(iv) Suppose $R_{1}, R_{2}$ are nondecreasing and $q$-varying with $q \in(0, \infty)$. Then, for $c \in(0, \infty)$, we have

$$
\lim _{u \rightarrow \infty} \frac{R_{1}(u)}{R_{2}(u)}=c \quad \text { if and only if } \quad \lim _{u \rightarrow \infty} \frac{R_{1}^{\leftarrow}(u)}{R_{2}^{\leftarrow}(u)}=c^{-1 / q}
$$

Proposition 2.6. (See also Proposition 4.10 in [Cîrstea and Trombetti 2008]). Let $R \in \mathbb{R V}_{q}$ and choose $B \geq 0$ so that $R$ is locally bounded on $[B, \infty)$. If $q>0$, then

(a) $\sup \{R(y): B \leq y \leq u\} \sim R(u)$ as $u \rightarrow \infty$,

(b) $\inf \{R(y): y \geq u\} \sim R(u)$ as $u \rightarrow \infty$.

If $q<0$, then

(c) $\inf \{R(y): y \geq u\} \sim R(u)$ as $u \rightarrow \infty$,

(d) $\inf \{R(y): B \leq y \leq u\} \sim R(u)$ as $u \rightarrow \infty$.

\section{Asymptotic properties of $\phi$}

Using Karamata's theory of regular variation and its extensions, we now consider the asymptotic properties of the function $\phi$ defined in (1-10).

Lemma 3.1. Let $m \in \mathfrak{K}_{\ell}$ and $f \in \mathbb{R} \mathbb{V}_{q}$ with $q>k$. If $\phi$ is defined by (1-10), then there exists a function $\psi \in C^{2}(0, \tau)$ with $\tau>0$ which satisfies $\lim _{t \rightarrow 0} \psi(t) / \phi(t)=1$ and

$$
\begin{aligned}
\lim _{t \rightarrow 0} \frac{\psi(t) \psi^{\prime \prime}(t)}{\left(\psi^{\prime}(t)\right)^{2}} & =1+\frac{(q-k) \ell}{k+1}, \\
\lim _{t \rightarrow 0} \frac{\left(-\psi^{\prime}(t)\right)^{k-1} \psi^{\prime \prime}(t)}{m^{k+1}(t) f(\psi(t))} & =\left(\frac{k+1}{q-k}\right)^{k+1}\left(1+\frac{(q-k) \ell}{k+1}\right) .
\end{aligned}
$$

where $\ell$ appears in (1-3). 
Proof. To prove (3-1), denote $g(u)=f(u) / u^{k}$. Since $g \in \mathbb{R} \mathbb{V}_{q-k}$ and $q>k$, by Proposition 2.6 we have $\lim _{u \rightarrow \infty} g(u) / \mathscr{P}(u)=1$. By Remark 4.8 in [Cîrstea and Trombetti 2008] we infer that there exists a function $\hat{g} \in C^{2}(0, \tau)$ such that $\lim _{u \rightarrow \infty} \hat{g}(u) / g(u)=1$ and

$$
\lim _{u \rightarrow \infty} \frac{u \hat{g}^{\prime}(u)}{\hat{g}(u)}=q-k, \quad \lim _{u \rightarrow \infty} \frac{u \hat{g}^{\prime \prime}(u)}{\hat{g}^{\prime}(u)}=q-k-1,
$$

where we have used $g \in \mathbb{R V}_{q-k}$.

We define $\psi$ by

$$
\hat{g}(\psi(t))=\left(\int_{0}^{t} m(s) d s\right)^{-k-1}, \quad \text { for } t>0 \text { small. }
$$

Notice that

$$
\phi(t)=\mathscr{P} \leftarrow\left(\left(\int_{0}^{t} m(s) d s\right)^{-k-1}\right), \quad \text { for } t>0 \text { small. }
$$

Thus Proposition 2.5 gives

$$
\lim _{t \rightarrow 0} \frac{\hat{g} \leftarrow\left(\left(\int_{0}^{t} m(s) d s\right)^{-k-1}\right)}{\mathscr{P} \leftarrow\left(\left(\int_{0}^{t} m(s) d s\right)^{-k-1}\right)}=\lim _{t \rightarrow 0} \frac{\hat{g}\left(\left(\int_{0}^{t} m(s) d s\right)^{-k-1}\right)}{\mathscr{P}\left(\left(\int_{0}^{t} m(s) d s\right)^{-k-1}\right)}=1,
$$

where we have used $\lim _{u \rightarrow \infty} g(u) / \mathscr{P}(u)=1$ and $\lim _{u \rightarrow \infty} \hat{g}(u) / g(u)=1$ in the last equality.

By the definition of the inverse of $\hat{g}$ we see that

$$
\lim _{t \rightarrow 0} \frac{\psi(t)}{\phi(t)}=\lim _{t \rightarrow 0} \frac{\hat{g}^{\leftarrow}\left(\left(\int_{0}^{t} m(s) d s\right)^{-k-1}\right)}{\mathscr{P} \leftarrow\left(\left(\int_{0}^{t} m(s) d s\right)^{-k-1}\right)}=1 .
$$

By differentiating (3-4) we obtain

$$
\hat{g}^{\prime}(\psi(t)) \psi^{\prime}(t)=-(k+1)\left(\int_{0}^{t} m(s) d s\right)^{-k-2} m(t), \quad \text { for } t>0 \text { small. }
$$

Then, by (3-3), (3-4) and (3-7),

$$
\frac{\psi^{\prime}(t)}{\psi(t)} \sim \frac{-(k+1)}{q-k} \frac{m(t)}{\int_{0}^{t} m(s) d s}, \quad \text { as } t \rightarrow 0 .
$$

We differentiate (3-7), then use (1-3) and (3-3) to deduce that as $t \rightarrow 0$

$$
\begin{aligned}
\hat{g}^{\prime}(\psi(t)) \frac{\left(\psi^{\prime}(t)\right)^{2}}{\psi(t)}(q-k-1 & \left.+\frac{\psi(t) \psi^{\prime \prime}(t)}{\left(\psi^{\prime}(t)\right)^{2}}\right) \\
& \sim(k+1)(k+1+\ell) m^{2}(s)\left(\int_{0}^{t} m(s) d s\right)^{-k-3}
\end{aligned}
$$


Putting (3-7) and (3-8) into (3-9), we have

$$
\begin{gathered}
-(k+1)\left(\int_{0}^{t} m(s) d s\right)^{-k-2} m(t) \frac{-(k+1)}{q-k} \frac{m(t)}{\int_{0}^{t} m(s) d s}\left(q-k-1+\frac{\psi(t) \psi^{\prime \prime}(t)}{\left(\psi^{\prime}(t)\right)^{2}}\right) \\
\quad=\frac{(k+1)^{2}}{q-k} m^{2}(t)\left(\int_{0}^{t} m(s) d s\right)^{-k-3}\left(q-k-1+\frac{\psi(t) \psi^{\prime \prime}(t)}{\left(\psi^{\prime}(t)\right)^{2}}\right) \\
\sim(k+1)(k+1+\ell) m^{2}(s)\left(\int_{0}^{t} m(s) d s\right)^{-k-3} .
\end{gathered}
$$

Thus,

$$
\frac{(k+1)}{q-k}\left(q-k-1+\frac{\psi(t) \psi^{\prime \prime}(t)}{\left(\psi^{\prime}(t)\right)^{2}}\right) \sim(k+1+\ell) .
$$

(3-1) now follows from (3-11).

From (3-4) and (3-8), we find

$$
\lim _{t \rightarrow 0}\left(-\frac{\psi^{\prime}(t)}{\psi(t)}\right)^{k+1} \frac{1}{m^{k+1}(t) \hat{g}(\psi(t))}=\left(\frac{k+1}{q-k}\right)^{k+1} .
$$

This, combined with (3-1), proves (3-2).

\section{Proof of Theorem 1.3}

Fix $\epsilon \in(0,1 / 2)$ and choose $\delta>0$ small enough such that:

(a) $m$ is nondecreasing on $(0,2 \delta)$.

(b) $\beta^{-}(1-\epsilon)(m(d(x)))^{k+1} \leq b(x) \leq \beta^{+}(1+\epsilon)(m(d(x)))^{k+1}$, for every $x \in \Omega_{2 \delta}$, where for $\lambda>0$ we set

$$
\Omega_{\lambda}=\{x \in \Omega: d(x)<\lambda\} .
$$

(c) $d(x)$ is a $C^{2}$ function on $\Gamma_{2 \delta}=\{x \in \bar{\Omega}: d(x)<2 \delta\}$.

(d) $0<\psi, \psi^{\prime}<0$, and $\psi^{\prime \prime}>0$ on $(0,2 \delta)$, where $\psi$ is as in Lemma 3.1.

(e) $\sigma_{k-1}\left(\operatorname{diag}\left(1-\rho_{1}(y) d(x), \ldots, 1-\rho_{n-1}(y) d(x)\right)\right)>1-\varepsilon$, for every $x \in \Omega_{2 \delta}$. Recall that $\rho_{i}(y), i=1, \ldots, n-1$, denote the principal curvatures of $\partial \Omega$ at $y$, where $y \in \partial \Omega$ is such that $|x-y|=d(x)$.

Fix $\tau \in(0, \delta)$. With $\xi^{ \pm}$given by (1-11), we set

$$
\eta^{ \pm}=((1 \mp \varepsilon)(1 \mp 2 \varepsilon))^{1 /(k-q)} \xi^{ \pm} .
$$

Define

$$
\begin{cases}v_{\tau}^{+}=\eta^{+} \psi\left(\left(1-e^{-T(d(x)-\tau)}\right) / T\right), & x \in \Omega_{2 \delta} \backslash \bar{\Omega}_{\tau}, \\ v_{\tau}^{-}=\eta^{-} \psi\left(\left(1-e^{-T(d(x)+\tau)}\right) / T\right), & x \in \Omega_{2 \delta-\tau} .\end{cases}
$$


Step 1. We prove that, near the boundary, $v_{\tau}^{+}$(respectively, $v_{\tau}^{-}$) is an upper (respectively, lower) solution of (1-1), that is,

$$
\begin{cases}H_{k}\left[D^{2} v_{\tau}^{+}\right] \leq b(x) f\left(v_{\tau}^{+}\right), & x \in \Omega_{2 \delta} \backslash \bar{\Omega}_{\tau}, \\ H_{k}\left[D^{2} v_{\tau}^{-}\right] \geq b(x) f\left(v_{\tau}^{-}\right), & x \in \Omega_{2 \delta-\tau} .\end{cases}
$$

We denote by

$$
M^{+}=\max _{y \in \partial \Omega} \sigma_{k-1}(y) \quad \text { and } \quad M^{-}=\min _{y \in \partial \Omega} \sigma_{k-1}(y) .
$$

After some computations we obtain, for a point $x \in \Omega_{2 \delta} \backslash \bar{\Omega}_{\tau}$,

$$
\left[v_{\tau}^{+}\right]_{i j}=\eta^{+} e^{-T(d(x)-\tau)}\left(\psi^{\prime} d_{i j}+d_{i} d_{j}\left(\psi^{\prime \prime} e^{-T(d(x)-\tau)}-T \psi^{\prime}\right)\right) .
$$

Since $|D d(x)|=1$ in $x \in \Omega_{2 \delta} \backslash \bar{\Omega}_{\tau}$, we can choose a coordinate system such that

$$
\begin{aligned}
\operatorname{Dd}(x) & =(0, \ldots, 0,1), \\
D^{2} d(x) & =\operatorname{diag}\left(d_{11}(x), \ldots, d_{n-1, n-1}(x), 0\right),
\end{aligned}
$$

where $d_{i i}(x)=-\rho_{i}(y) /\left(1-\rho_{i}(y) d(x)\right)$, and $y \in \partial \Omega$ is such that $|x-y|=d(x)$ as in Corollary 2.3.

Hence

$$
D^{2} v_{\tau}^{+}=\eta^{+} e^{-T(d(x)-\tau)} \operatorname{diag}\left(\psi^{\prime} d_{11}(x), \ldots, \psi^{\prime} d_{n-1, n-1}(x), \psi^{\prime \prime} e^{-T(d(x)-\tau)}-T \psi^{\prime}\right) .
$$

Using this and Corollary 2.3, we can easily compute the $k$-Hessian of $v_{\tau}^{+}$:

$$
\begin{aligned}
H_{k}\left[D^{2} v_{\tau}^{+}\right] & =\left(\eta^{+}\right)^{k} e^{-(k+1) T(d(x)-\tau)}\left[-\psi^{\prime}\right]^{k-1} \psi^{\prime \prime} \sigma_{k-1}\left(-D^{2} d(x)\right) \\
+ & \left(\eta^{+}\right)^{k} e^{-k T(d(x)-\tau)}\left[-\psi^{\prime}\right]^{k}\left(T \sigma_{k-1}\left(-D^{2} d(x)\right)+\sigma_{k}\left(-D^{2} d(x)\right)\right)
\end{aligned}
$$

Now, if

$$
T_{1} \leq-\frac{\max _{\Omega_{2 \delta} \backslash \bar{\Omega}_{\tau}}\left|\sigma_{k}\left(D^{2} d(x)\right)\right|}{\min _{\Omega_{2 \delta} \backslash \bar{\Omega}_{\tau}} \sigma_{k-1}\left(-D^{2} d(x)\right)},
$$

then (4-5) and condition (e) yield for $T \leq T_{1}$,

$$
\begin{aligned}
H_{k}\left[D^{2} v_{\tau}^{+}\right] & \leq\left(\eta^{+}\right)^{k} e^{-(k+1) T(d(x)-\tau)}\left[-\psi^{\prime}\right]^{k-1} \psi^{\prime \prime} \sigma_{k-1}\left(-D^{2} d(x)\right), \\
& \leq \frac{\left(\eta^{+}\right)^{k}}{1-\varepsilon} M^{+} e^{-(k+1) T(d(x)-\tau)}\left[-\psi^{\prime}\right]^{k-1} \psi^{\prime \prime}, x \in \Omega_{2 \delta} \backslash \bar{\Omega}_{\tau} .
\end{aligned}
$$

Similarly, we have for $T_{2}$

$$
T_{2} \geq \frac{\max _{\Omega_{2 \delta-\tau}}\left|\sigma_{k}\left(D^{2} d(x)\right)\right|}{\min _{\Omega_{2 \delta-\tau}} \sigma_{k-1}\left(-D^{2} d(x)\right)},
$$


for $T \geq T_{2}$,

$$
\begin{aligned}
H_{k}\left[D^{2} v_{\tau}^{-}\right] & \geq\left(\eta^{-}\right)^{k} e^{-(k+1) T(d(x)+\tau)}\left[-\psi^{\prime}\right]^{k-1} \psi^{\prime \prime} \sigma_{k-1}\left(-D^{2} d(x)\right), \\
& \geq \frac{\left(\eta^{-}\right)^{k}}{1+\varepsilon} M^{-} e^{-(k+1) T(d(x)+\tau)}\left[-\psi^{\prime}\right]^{k-1} \psi^{\prime \prime}, \quad x \in \Omega_{2 \delta-\tau} .
\end{aligned}
$$

Therefore, to deduce (4-3) it is enough to establish that

$$
\lim _{t \rightarrow 0}\left(\eta^{ \pm}\right)^{k} \frac{M^{ \pm}}{\beta^{\mp}} \frac{\left[-\psi^{\prime}(t)\right]^{k-1} \psi^{\prime \prime}(t)}{m^{k+1}(t) f\left(\eta^{ \pm} \psi(t)\right)}=(1 \mp \varepsilon)(1 \mp \varepsilon) .
$$

Since $f \in \mathbb{R V}_{q}$, Lemma 3.1 and our choice of $\eta^{ \pm}$in (4-1),

$$
\begin{gathered}
\lim _{t \rightarrow 0}\left(\eta^{ \pm}\right)^{k} \frac{M^{ \pm}}{\beta^{\mp}} \frac{\left[-\psi^{\prime}(t)\right]^{k-1} \psi^{\prime \prime}(t)}{m^{k+1}(t) f\left(\eta^{ \pm} \psi(t)\right)}=\left(\eta^{ \pm}\right)^{k} \frac{M^{ \pm}}{\beta^{\mp}}\left(\frac{k+1}{q-k}\right)^{k+1}\left(1+\frac{(q-k) \ell}{k+1}\right)\left(\eta^{ \pm}\right)^{-q} \\
=((1 \mp \varepsilon)(1 \mp 2 \varepsilon)) \xi^{ \pm(k-q)} \frac{M^{ \pm}}{\beta^{\mp}}\left(\frac{k+1}{q-k}\right)^{k+1}\left(1+\frac{(q-k) \ell}{k+1}\right)=(1 \mp \varepsilon)(1 \mp 2 \varepsilon),
\end{gathered}
$$

where we have used (1-11) in the last equality.

Step 2. Every strictly $k$-convex blow-up solution u of (1-1) satisfies (1-9).

Let $C=\max _{d(x)=\delta} u(x)$. Notice that

$$
\begin{cases}v_{\tau}^{+}+C=\infty>u(x), & x \in \Omega \text { with } d(x)=\tau, \\ v_{\tau}^{+}+C \geq u(x), & x \in \Omega \text { with } d(x)=\delta .\end{cases}
$$

Using (4-3) we deduce that for every $x \in \Omega_{\delta} \backslash \bar{\Omega}_{\tau}$,

$$
H_{k}\left[D^{2}\left(v_{\tau}^{+}+C\right)\right]=H_{k}\left[D^{2} v_{\tau}^{+}\right] \leq b(x) f\left(v_{\tau}^{+}\right) \leq b(x) f\left(v_{\tau}^{+}+C\right) .
$$

Since $u$ is a solution to (1-1), by the comparison principle for $k$-Hessians [Jian 2006, Lemma 2.1] we find

$$
v_{\tau}^{+}+C \geq u(x), \quad \text { for all } x \in \Omega_{\delta} \backslash \bar{\Omega}_{\tau} .
$$

We set $C^{\prime}=\xi^{-} \psi(\delta)$. Hence, we have $C^{\prime} \geq v_{\tau}^{-}(x)$ for every $x \in \Omega$ with $d(x)=\delta-\tau$. It follows that

$$
u(x)+C^{\prime} \geq v_{\tau}^{-}(x), \quad \text { for all } x \in \partial \boldsymbol{\Omega}_{\delta-\tau} .
$$

We see that, for every $x \in \Omega_{\delta-\tau}$,

$$
H_{k}\left[u(x)+C^{\prime}\right]=H_{k}\left[D^{2} u(x)\right] \leq b(x) f(u(x)) \leq b(x) f\left(u(x)+C^{\prime}\right),
$$

while by (4-3) we have

$$
H_{k}\left[D^{2} v_{\tau}^{-}\right] \geq b(x) f\left(v_{\tau}^{-}\right), \quad x \in \Omega_{\delta-\tau} .
$$


Using again the comparison principle for $k$-Hessian equations, we infer that

$$
u(x)+C^{\prime} \geq v_{\tau}^{-}(x), \quad \text { for all } x \in \Omega_{\delta-\tau} .
$$

By (4-8) and (4-11), letting $\tau \rightarrow 0$ we obtain

$$
\left\{\begin{array}{l}
((1+\epsilon)(1+2 \epsilon))^{1 /(k-q)} \xi^{-} \psi\left(\left(1-e^{-T_{2} d(x)}\right) / T_{2}\right)-C^{\prime} \leq u(x), x \in \Omega_{\delta} \\
u(x) \leq((1-\epsilon)(1-2 \epsilon))^{1 /(k-q)} \xi^{+} \psi\left(\left(1-e^{-T_{1} d(x)}\right) / T_{1}\right)+C
\end{array}\right.
$$

Dividing by $\psi\left(\left(1-e^{-T_{i} d(x)}\right) / T_{i}\right)$ for $i=1,2$ and noticing that $\lim _{t \rightarrow 0} \psi(t) / \phi(t)=$ 1 , letting $d(x) \rightarrow 0$, we obtain

$$
\left\{\begin{array}{l}
\liminf _{d(x) \rightarrow 0} u / \phi(d(x)) \geq((1+\epsilon)(1+2 \epsilon))^{1 /(k-q)} \xi^{-}, \\
\liminf _{d(x) \rightarrow 0} u / \phi(d(x)) \leq((1-\epsilon)(1-2 \epsilon))^{1 /(k-q)} \xi^{+} .
\end{array}\right.
$$

Since $\epsilon>0$ is arbitrary, we let $\epsilon \rightarrow 0$ and obtain (1-9). This completes the proof of Theorem 1.3.

\section{Proof of Theorem 1.6}

We follow the methods in [Cîrstea and Trombetti 2008] and divide the proof into two steps:

Step 1. For all strictly $k$-convex blow-up solutions $u_{1}, u_{2}$ of (1-1),

$$
\lim _{d(x) \rightarrow 0} \frac{u_{1}(x)}{u_{2}(x)}=1 .
$$

Step 2. There is at most one strictly convex blow-up solution of (1-1).

Proof of Step 1. The argument breaks into two cases.

Case (i): $b>0$ on $\bar{\Omega}$. Since $u_{1}$ and $u_{2}$ are arbitrary, it suffices to show that

$$
\liminf _{d(x) \rightarrow 0} \frac{u_{1}(x)}{u_{2}(x)} \geq 1 .
$$

Without loss of generality, we can assume that 0 belongs to $\Omega$. Let $\varepsilon \in(0,1)$ be fixed and let $\lambda>1$ be close to 1 .

We set

$$
C_{\lambda}=\left((1+\varepsilon) \lambda^{2 k} \max _{x \in(1 / \lambda) \bar{\Omega}} \frac{b(\lambda x)}{b(x)}\right)^{1 /(q-k)},
$$

where $(1 / \lambda) \bar{\Omega}=\{(1 / \lambda) x: x \in \bar{\Omega}\}$. Notice that $C_{\lambda} \rightarrow(1+\varepsilon)^{1 /(q-k)}$ as $\lambda \rightarrow 1$.

Hence, by Proposition 2.4 and $\lim _{d(x) \rightarrow 0} u_{1}(x)=\infty$, we deduce that there exists $\delta=\delta(\varepsilon)>0$, independent of $\lambda$, such that

(5-4) $C_{\lambda}^{q} \frac{f\left(u_{1}(x)\right)}{f\left(C_{\lambda} u_{1}(x)\right)} \leq 1+\varepsilon, \quad$ for all $x \in \Omega_{\delta}$ and $\lambda \in(1,1+\eta)$ for some $\eta$. 
We now define $U_{\lambda}$ as

$$
U_{\lambda}(x)=C_{\lambda} u_{1}(\lambda x), \quad \text { for all } x \in(1 / \lambda) \Omega_{\delta} .
$$

Notice by (5-3)-(5-5),

$$
\begin{aligned}
H_{k}\left[D^{2} U_{\lambda}(x)\right] & =\lambda^{2 k} C_{\lambda}^{k} b(\lambda x) f\left(u_{1}(\lambda x)\right) \\
& \leq \lambda^{2 k} C_{\lambda}^{k-q}(1+\varepsilon) b(\lambda x) f\left(C_{\lambda} u_{1}(\lambda x)\right) \\
& \leq b(x) f\left(C_{\lambda} u_{1}(\lambda x)\right)=b(x) f\left(U_{\lambda}(x)\right), \quad x \in(1 / \lambda) \Omega_{\delta},
\end{aligned}
$$

which says that $U_{\lambda}(x)$ is a supersolution of (1-1) with domain $(1 / \lambda) \Omega_{\delta}$.

Since $f$ is increasing on $(0, \infty)$ and $(5-6)$, for each constant $M>0$,

$$
\begin{aligned}
H_{k}\left[D^{2}\left(U_{\lambda}(x)+M\right)\right] & =H_{k}\left[D^{2} U_{\lambda}(x)\right] \leq b(x) f\left(U_{\lambda}(x)\right) \\
& \leq b(x) f\left(U_{\lambda}(x)+M\right), \quad \text { for all } x \in(1 / \lambda) \Omega_{\delta} .
\end{aligned}
$$

Notice also that $U_{\lambda}(x)=\infty>u_{2}(x)$, for every $x \in(1 / \lambda) \partial \Omega$. Moreover, $x \in$ $(1 / \lambda) \partial \Omega$ implies that $d(x)<\delta$ (as $\lambda>1$ is close to 1 ).

Thus, if we choose $M>0$ large enough (for example, $M=\max _{d(x)=\delta} u_{2}(x)$ ), then by the comparison principle for $k$-Hessian equations we obtain

$$
U_{\lambda}(x)+M \geq u_{2}(x), \quad \text { for all } x \in \Omega_{\delta} \cap(1 / \lambda) \Omega_{\delta} .
$$

Letting $\lambda \rightarrow 1$ in (5-8), we find

$$
(1+\varepsilon)^{1 /(q-k)} u_{1}(x)+M \geq u_{2}(x), \quad \text { for all } x \in \Omega_{\delta},
$$

which implies that

$$
\liminf _{d(x) \rightarrow 0} \frac{u_{1}(x)}{u_{2}(x)} \geq(1+\varepsilon)^{1 /(k-q)},
$$

and then letting $\varepsilon \rightarrow 0$ we obtain (5-2).

Case (ii): $b \equiv 0$ on $\partial \Omega, \Omega$ is a ball of radius $R>0$, and (1-13) holds. By Corollary 1.5 , every strictly $k$-convex blow-up solution $u$ of (1-1) satisfies

$$
\lim _{d(x) \rightarrow 0} \frac{u}{\phi(d(x))}=\left(\frac{((q-k) /(k+1))^{k+1} R^{k-1}}{1+\ell(q-k) /(k+1)}\right)^{1 /(k-q)},
$$

where $\phi$ is defined by (1-10) and $\ell$ appears in (1-3).

Proof of Step 2. If $u_{1}, u_{2}$ are arbitrary strictly $k$-convex blow-up solutions of (1-1), it suffices to show that $u_{1} \leq u_{2}$ in $\Omega$. Fix $\varepsilon>0$. By Step 1 we infer that

$$
\lim _{d(x) \rightarrow 0}\left(u_{1}(x)-(1+\varepsilon) u_{2}(x)\right)=-\infty .
$$


Since $f(u) / u^{k}$ is increasing on $(0, \infty)$, we deduce that

$$
\begin{aligned}
H_{k}\left[D^{2}(1+\varepsilon) u_{2}(x)\right] & =(1+\varepsilon)^{k} H_{k}\left[D^{2} u_{2}(x)\right] \leq(1+\varepsilon)^{k} b(x) f\left(u_{2}(x)\right) \\
& \leq b(x) f\left((1+\varepsilon) u_{2}(x)\right), \quad \text { for all } x \in \Omega .
\end{aligned}
$$

By (5-12), (5-13) and the comparison principle for $k$-Hessian equations,

$$
u_{1} \leq(1+\varepsilon) u_{2}, \quad \text { for all } x \in \Omega .
$$

Letting $\varepsilon \rightarrow 0$, thus $u_{1} \leq u_{2}$ in $\Omega$. This completes the proof of Step 2 and hence of Theorem 1.6.

\section{Acknowledgments}

We thank Professor Huaiyu Jian for his encouragement and many suggestions in this project, and Doctor Lu Xu for helpful discussions.

\section{References}

[Bandle and Marcus 1992] C. Bandle and M. Marcus, “"Large” solutions of semilinear elliptic equations: existence, uniqueness and asymptotic behaviour", J. Anal. Math. 58 (1992), 9-24. MR 94c:35081 Zbl 0802.35038

[Bandle and Marcus 1995] C. Bandle and M. Marcus, "Asymptotic behaviour of solutions and their derivatives, for semilinear elliptic problems with blowup on the boundary", Ann. Inst. H. Poincaré Anal. Non Linéaire 12:2 (1995), 155-171. MR 96e:35038 Zbl 0840.35033

[Caffarelli et al. 1985] L. Caffarelli, L. Nirenberg, and J. Spruck, "The Dirichlet problem for nonlinear second-order elliptic equations. III. Functions of the eigenvalues of the Hessian”, Acta Math. 155:3-4 (1985), 261-301. MR 87f:35098 Zbl 0654.35031

[Chuaqui et al. 2004] M. Chuaqui, C. Cortazar, M. Elgueta, and J. Garcia-Melian, "Uniqueness and boundary behavior of large solutions to elliptic problems with singular weights", Commun. Pure Appl. Anal. 3:4 (2004), 653-662. MR 2005h:35115 Zbl 02143210

[Cîrstea and Rădulescu 2006] F. C. Cîrstea and V. Rădulescu, "Nonlinear problems with boundary blow-up: a Karamata regular variation theory approach", Asymptot. Anal. 46:3-4 (2006), 275-298. MR 2007a:35045 Zbl 05042405

[Cîrstea and Trombetti 2008] F. C. Cîrstea and C. Trombetti, "On the Monge-Ampère equation with boundary blow-up: existence, uniqueness and asymptotics", Calc. Var. Partial Differential Equations 31:2 (2008), 167-186. MR 2008k:35178 Zbl 1148.35022

[Colesanti et al. 2000] A. Colesanti, P. Salani, and E. Francini, "Convexity and asymptotic estimates for large solutions of Hessian equations", Differential Integral Equations 13:10-12 (2000), 14591472. MR 2001j:35075 Zbl 0977.35046

[García-Melián 2006] J. García-Melián, "Nondegeneracy and uniqueness for boundary blow-up elliptic problems", J. Differential Equations 223:1 (2006), 208-227. MR 2007b:35113 Zbl 05019771

[García-Melián et al. 2001] J. García-Melián, R. Letelier-Albornoz, and J. Sabina de Lis, "Uniqueness and asymptotic behaviour for solutions of semilinear problems with boundary blow-up", Proc. Amer. Math. Soc. 129:12 (2001), 3593-3602. MR 2002j:35117 Zbl 0989.35044

[Gilbarg and Trudinger 1998] D. Gilbarg and N. S. Trudinger, Elliptic partial differential equations of second order, 2nd ed., Springer, Berlin, 1998. MR 2001k:35004 Zbl 1042.35002 
[Guan and Jian 2004] B. Guan and H.-Y. Jian, "The Monge-Ampère equation with infinite boundary value", Pacific J. Math. 216:1 (2004), 77-94. MR 2005f:35100 Zbl 1126.35318

[Jian 2006] H. Jian, "Hessian equations with infinite Dirichlet boundary value", Indiana Univ. Math. J. 55:3 (2006), 1045-1062. MR 2008f:35120 Zbl 1126.35026

[Mohammed 2007] A. Mohammed, "On the existence of solutions to the Monge-Ampère equation with infinite boundary values", Proc. Amer. Math. Soc. 135:1 (2007), 141-149. MR 2008f:35129 Zbl 05120216

[Osserman 1957] R. Osserman, "On the inequality $\Delta u \geq f(u)$ ", Pacific J. Math. 7 (1957), 16411647. MR 20 \#4701 Zbl 0083.09402

[Resnick 1987] S. I. Resnick, Extreme values, regular variation, and point processes, Applied Probability 4, Springer, New York, 1987. MR 89b:60241 Zbl 0633.60001

[Salani 1998] P. Salani, "Boundary blow-up problems for Hessian equations", Manuscripta Math. 96:3 (1998), 281-294. MR 99e:35071 Zbl 0907.35052

[Takimoto 2006] K. Takimoto, "Solution to the boundary blowup problem for $k$-curvature equation", Calc. Var. Partial Differential Equations 26:3 (2006), 357-377. MR 2007h:35115 Zbl 1105.35039

[Trudinger 1995] N. S. Trudinger, "On the Dirichlet problem for Hessian equations", Acta Math. 175:2 (1995), 151-164. MR 96m:35113 Zbl 0887.35061

[Véron 1992] L. Véron, "Semilinear elliptic equations with uniform blow-up on the boundary", $J$. Anal. Math. 59 (1992), 231-250. MR 94k:35113 Zbl 0802.35042

Received September 18, 2008. Revised February 3, 2009.

\author{
YONG HUANG \\ Wuhan Institute of Physics And Mathematics \\ Chinese ACAdemy of Sciences \\ WUHAN 430071 \\ CHINA \\ huangyong@wipm.ac.cn
}

